Pacific Journal of Mathematics

FINITE GROUPS WITH SMALL CHARACTER DEGREES AND

1. Martin (IRVing) ISAac and Donald SteVen Passim 


\title{
FINITE GROUPS WITH SMALL CHARACTER DEGREES AND LARGE PRIME DIVISORS II
}

\author{
I. M. Isaacs and D. S. Passman
}

In a previous paper one of the authors considered groups $G$ with r. b. $n$ (representation bound $n$ ) and $n<p^{2}$ for some prime $p$. Here we continue this study. We first offer a new proof of the fact that if $n=p-1$ then $G$ has a normal Sylow $p$ subgroup. Next we show that if $n=p^{3 / 2}$ then $p^{2} \nmid\left|G / O_{p}(G)\right|$. Finally we consider $n=2 p-1$ and with the help of the modular theory we obtain a fairly precise description of the structure of $G$. In particular we show that its composition factors are either p-solvable or isomorphic to $P S L(2, p), P S L(2$, $p-1)$ for $p$ a Fermat prime or $P S L(2, p+1)$ for $p$ a Mersenne prime.

Now the irreducible characters of $\operatorname{PSL}(2, \mathrm{p})$ have degrees (see [10] p. 128) $1, p, p \pm 1,(p \pm 1) / 2$ for $p$ odd and those of $P S L\left(2,2^{a}\right)$ have degrees (see [10] p. 134) $1,2^{a}, 2^{a} \pm 1$. Thus for $p>2$ the linear groups of the preceding paragraph do in fact have r.b. $(2 p-1)$.

The notation here is standard. In addition, if $\chi$ is a character of $G$ we let det $\chi$ denote the linear character which is the determinant of the representation associated with $\chi$. Also $n_{p}(G)$ denotes the number of Sylow $p$-subgroups of $G$.

Lemma 1. Let $G$ be a group with r.b.n. and let $N \neq G$ be a subgroup. Suppose $G=\bigcup_{i=0}^{t} N x_{i} N$ is the $(N, N)$-double coset decomposition of $G$ with $x_{0}=1$. Set $a_{i}=\left|N x_{i} N\right| /|N|=\left[N: N \cap N^{x_{i}}\right]$. Then $n \geqq\left(a_{1}+a_{2}+\cdots+a_{t}\right) / t$.

Proof. Let $\theta=\left(1_{N}\right)^{G}$ be the character of the permutation representation of $G$ on the cosets of $N$. Then $\theta(1)=[G: N],\left[\theta, 1_{G}\right]=1$ and $\|\theta\|^{2}=1+t$. Since $\left[\theta, 1_{G}\right]=1$ we can write

$$
\theta=1_{G}+b_{1} \chi_{1}+\cdots+b_{s} \chi_{s}
$$

where the $\chi_{i}$ are distinct nonprincipal irreducible characters of $G$. Thus since $G$ has r.b.n we have

$$
\begin{aligned}
1+n t & =1+n\left(\|\theta\|^{2}-1\right)=1+n\left(b_{1}^{2}+\cdots+b_{s}^{2}\right) \\
& \geqq 1+n\left(b_{1}+\cdots+b_{s}\right) \geqq 1+b_{1} \chi_{1}(1)+\cdots+b_{s} \chi_{s}(1) \\
& =\theta(1)=1+\left(a_{1}+a_{2}+\cdots+a_{t}\right)
\end{aligned}
$$

and the result follows. 
LEMma 2. Let $G$ be a group with r.b.n.

(i) Let $N \neq G$ be a subgroup. Then

$$
n \geqq \min \left\{\left[N: N \cap N^{x}\right] \mid x \in G-N\right\} \text {. }
$$

(ii) Let $\pi$ be a set of primes and let $H$ be a maximal $\pi$-subgroup of $G$. Then either $H \triangle G$ or $n \geqq \min \left\{\left[H: H \cap H^{x}\right] \mid x \in G-N(H)\right\}$.

Proof. (i) follows immediately from Lemma 1. Now let $H$ be as in (ii) and suppose $H$ is not normal in $G$. Set $N=N(H) \neq G$. Since $H$ is a maximal $\pi$-subgroup it follows that $H=O_{\pi}(N)$. Thus if $x \in G$ then $H^{x}=\boldsymbol{O}_{\pi}\left(N^{x}\right)$ so $H \cap N^{x}=H \cap H^{x}$ and

$$
\left[N: N \cap N^{x}\right] \geqq\left[H: H \cap N^{x}\right]=\left[H: H \cap H^{x}\right] .
$$

Thus the result follows from (i).

Applying Lemma 2(ii) with $\pi=\{p\}$ and $H$ a Sylow $p$-subgroup of $G$ yields

THEOREM 3. Let $p$ be a prime and let $G$ be a group with r.b. $(p-1)$. Then $n_{p}(G)=1$.

This result was originally proved in [7] (Theorem E) in a much more complicated way.

Lemma 4. Let $G$ have r.b. $\left(p^{2}-1\right)$ and let $Q_{1}$ and $Q_{2}$ be $p$-subgroups of $G$ with $\left\langle Q_{1}, Q_{2}\right\rangle$ not a p-group. Then $n_{p}\left(C\left(Q_{1}\right) \cap C\left(Q_{2}\right)\right)=1$. If further the Sylow p-subgroups of $G$ are abelian, then

$$
n_{p}\left(N\left(Q_{1}\right) \cap N\left(Q_{2}\right)\right)=1
$$

Proof. Set $W=\left\langle Q_{1}, Q_{2}\right\rangle$. Since $W$ is not a $p$-group we see that $n_{p}(W)>1$. We assume now that $n_{p}(C)>1$ where $C=\boldsymbol{C}\left(Q_{1}\right) \cap \boldsymbol{C}\left(Q_{2}\right)=$ $C(W)$ and derive a contradiction. Set $Z=W \cap C$ so that $Z$ is central in $W$ and $C$ and let $\bar{W}=W / Z, \bar{C}=C / Z$. Since $Z$ is central we have easily $n_{p}(\bar{W})>1, n_{p}(\bar{C})>1$ and $(W C) / Z=\bar{W} \times \bar{C}$. By Theorem 3 both $\bar{W}$ and $\bar{C}$ have irreducible characters of degree $\geqq p$ and hence $\bar{W} \times \bar{C}$ has an irreducible character of degree $\geqq p^{2}$. This is a contradiction since $G$ has r.b. $\left(p^{2}-1\right)$ and this property is inherited by subgroups and quotient groups. If the Sylow $p$-subgroups of $G$ are abelian then any $p$-group normalizing $Q_{i}$ centralizes it. Thus the second result follows from the first.

Theorem 5. Let $p$ be a prime and let $G$ be a group with r.b.p.p. Then $p^{2} \nmid\left|G / O_{p}(G)\right|$. 
Proof. If $p=2$ then $G$ has r.b.2 and the result follows from Theorem $\mathrm{C}$ of [7]. Thus we can assume that $p \geqq 3$ and clearly also that $\boldsymbol{O}_{p}(G)=\langle 1\rangle$. Since $p^{2}-p-1 \geqq\left[p^{3 / 2}\right]$ for $p \geqq 3$, Proposition 1.3 of [6] implies that a Sylow $p$-subgroup $P$ of $G$ is abelian. We assume that $|P| \geqq p^{2}$ and derive a contradiction. Set $n=p^{3 / 2}$.

Let $N=N(P)$ so that $N \neq G$. By Lemma 2(i) there exists $w \in G-N$ with $n \geqq\left[N: N \cap N^{w}\right] \geqq\left[P: P \cap P^{w}\right]$. Set $Q=P \cap P^{w}$ so since $p^{2}>n$ and $w \notin N$ we see that $[P: Q]=p$ and hence $Q \neq\langle 1\rangle$. Let $M=N(Q)$. Since $P \triangle N, P^{w} \triangle N^{w}$ we have $Q \triangle\left(N \cap N^{w}\right)$. Also $Q \triangle P$ and $P \nsubseteq N^{w}$. Hence $M \cap N \supseteqq\left\langle P, N \cap N^{w}\right\rangle$ so $[N: N \cap M] \leqq$ $n / p=p^{1 / 2}$.

We now make the following crucial observation. If $\left[M: M \cap M^{x}\right]<$ $p^{2}$ for some $x \notin M$ then $Q$ and $Q^{x}$ commute elementwise and $x \in M N M$. To see this suppose that $Q$ and $Q^{x}$ do not commute. Then since the Sylow $p$-subgroups of $G$ are abelian, $\left\langle Q, Q^{x}\right\rangle$ is not a $p$-group. By Lemma $4, n_{p}\left(M \cap M^{x}\right)=1$ so if $U=\boldsymbol{O}_{p}\left(M \cap M^{x}\right)$ then $U$ is also a Sylow $p$-subgroup of $M \cap M^{x}$. Now $p^{2} \nmid\left[M: M \cap M^{x}\right]$ and $Q \nsubseteq M^{x}$ clearly so $Q U$ is a Sylow $p$-subgroup of $M$. Since $N_{M}(Q U) \supseteqq\langle Q$, $\left.M \cap M^{x}\right\rangle$ we have $\left[M: N_{M}(Q U)\right]<p$ and hence by Sylow's theorem $Q U \triangle M$ and $n_{p}(M)=1$. This is a contradiction since $Q=P \cap P^{w}$ and $P, P^{w} \subseteq M$. Thus $Q$ and $Q^{x}$ commute and since $Q \neq Q^{x}$ and $[P: Q]=p$ it follows that $Q Q^{x}=P^{y^{-1}}$ is a Sylow $p$-subgroup of $G$. Thus $Q, Q^{y}$ and $Q^{x y}$ are all contained in $P$. By Burnside's lemma these three groups are conjugate in $N$. Thus $Q^{y}=Q^{h}, Q^{x y}=Q^{k}$ for some $h, k \in N$. This yields $y h^{-1} \in M, x y k^{-1} \in M$ so

$$
x=\left(x y k^{-1}\right) k h^{-1}\left(y h^{-1}\right)^{-1} \in M N M .
$$

Since $Q \neq\langle 1\rangle$ we have $M \neq G$. Let $G=\bigcup_{i=0}^{t} M x_{i} M$ be the $(M, M)$ double coset decomposition of $G$ with $x_{0}=1$. Set $a_{i}=\left|M x_{i} M\right| /|M|$ and suppose that there are precisely $r$ such $i \neq 0$ with $a_{i}<p^{2}$ and $s$ with $a_{i} \geqq p^{2}$. Then by Lemma $1, p^{3 / 2}=n \geqq\left(r+p^{2} s\right) /(r+s)$. Clearly $r \neq 0$ here so

$$
p^{3 / 2} \geqq\left(r+p^{2} s\right) /(r+s)>p^{2} /(1+r / s) .
$$

If $s=0$ then by the preceding paragraph $Q$ commutes with all its conjugates. This implies that $\left\langle Q^{x} \mid x \in G\right\rangle$ is a nontrivial normal $p$ subgroup of $G$, a contradiction. Thus $s \geqq 1$. Also if $a_{i}<p^{2}$ then $M x_{i} M \leqq M N M$ by the above. Since we have seen that $[N: N \cap M] \leqq$ $p^{1 / 2}$ we have $r \leqq p^{1 / 2}-1$ since the double coset $M$ itself is not counted. Thus $r / s \leqq p^{1 / 2}-1$ and

$$
p^{3 / 2}>p^{2} /(1+r / s) \geqq p^{3 / 2}
$$

a contradiction and the result follows. 
We now turn to the main result of this paper.

Theorem 6. Let $p$ be a prime and let $G$ be a nonabelian simple group with r.b. $(2 p-1)$. Then $p>2$ and we have one of the following.

(i) $G$ is a $p^{\prime}$-group.

(ii) $G \cong P S L(2, p)$ for $p>3$.

(iii) $G \cong P S L(2, p-1)$ for $p$ a Fermat prime, $p>3$.

(iv) $G \cong P S L(2, p+1)$ for $p$ a Mersenne prime.

Proof. Since groups with r.b.3 are solvable (Corollary 6.5 of [8]) we have $p>2$. By Theorem 5 since $p^{3 / 2} \geqq 2 p-1$ for $p>2$ we have $p^{2} \nmid|G|$. If $p \nmid|G|$ then $G$ satisfies (i) above. Thus we can assume now that $G$ has a Sylow $p$-subgroup $P$ of order $p$. Let $B_{1}(p)$ denote the principal $p$-block of $G$. We will use freely the structure of $B_{1}(p)$ and its associated tree as described in [1] and [2]. Since $B_{1}(p)$ contains a nonprincipal irreducible character $\chi$ and $\chi(1)<2 p$, Lemma 1 of [3] implies that $P$ is self centralizing. Let $N=N(P)$ and let $e=|N / P|$ so that $e \mid(p-1)$. By Burnside's transfer theorem $e>1$. We assume now that $G \not P S L(2, p)$ and $G \not P S L(2, p-1)$ for $p$ a Fermat prime.

Step 1. Let $\theta$ denote an exceptional character in $B_{1}(p)$. Then the tree of $B_{1}(p)$ must be one of the following.

$$
\begin{aligned}
& \text { (1) } \quad e=2 \quad 1_{G} \circ \begin{array}{cc}
\theta & \chi_{1} \\
p+2 & p+1
\end{array} \\
& \text { (2) } \quad e=2 \quad 1_{G} \circ \frac{\chi_{1}}{2 p-1} \stackrel{\theta}{\circ}{ }^{\circ}-2
\end{aligned}
$$

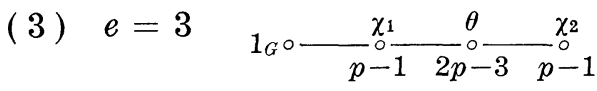

$$
\begin{aligned}
& \text { (4) } e=4
\end{aligned}
$$

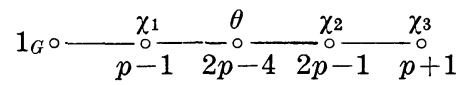

$$
\begin{aligned}
& \text { (5) } e=4
\end{aligned}
$$

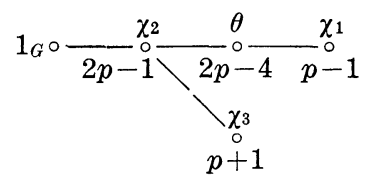

$$
\begin{aligned}
& \text { (6) } e=5
\end{aligned}
$$

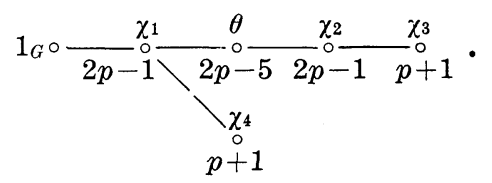

Here the degree of the character is written below the character designation.

Since $G$ is nonabelian and simple it follows that every nonprincipal irreducible representation of $G$ is faithful. Thus by Theorem 2 of [5] the degrees of the nonprincipal ordinary irreducible characters of $G$ are all $\geqq p-1$ and by Theorem 1 of [4] the degrees of the nonprincipal 
irreducible Brauer characters of $G$ are $\geqq 2(p-1) / 3$. Now $G$ has r.b. $(2 p-1)$ and the degrees of the ordinary irreducible characters of $B_{1}(p)$ satisfy $\chi(1) \equiv \pm 1(p)$ if $\chi$ is nonexceptional and $\theta(1) \equiv \pm e(p)$. This yields easily $\chi(1)=p-1, p+1,2 p-1$ or $\chi=1_{G}$ and $\theta(1)=$ $2 p-e, p+e$ or $\theta(1)=e$ if $e=p-1$.

Suppose first that $p=3$. Since $e \mid(p-1)$ we have $e=2$ and the tree is a line with three vertices. Now the center degree is maximal and the principal character must occur so we have clearly

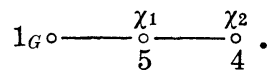

This is either tree (1) or tree (2) according to which of $\chi_{1}$ of $\chi_{2}$ we consider exceptional.

Now assume that $p \geqq 5$. Let $\chi \in B_{1}(p)$ with $\chi(1)=p-1$ and suppose that $\chi$ is modular reducible. We can denote this latter fact graphically by

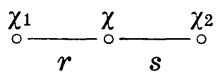

where $r$ and $s$ are degrees of modular constituents. Since $r+s \leqq$ $p-1$ we cannot have both $r, s \geqq 2(p-1) / 3$. This implies that one of the modular constituents is the principal character and the other has degree $p-2$.

Now let $\chi \in B_{1}(p)$ with $\chi(1)=p+1$ and suppose $\chi$ is modular reducible. Say we have

$$
\underset{\circ}{\chi_{1}} \frac{\chi}{r} \stackrel{\chi_{2}}{s}
$$

Here by the alternating nature of the tree $\chi_{1}, \chi_{2} \neq 1_{G}$ so $r, s \geqq$ $2(p-1) / 3$. Since $r+s \leqq p+1$ this yields $4(p-1) / 3 \leqq p+1$ so $p=$ 5 or 7. In fact either $p=5, r=s=3$ or $p=7, r=s=4$. We consider these in turn. Let $p=5$. Since $e \mid(p-1)$ we have $e=2$ or 4 . Certainly $e \neq 2$ here since $B_{1}(p)$ contains $e+1$ ordinary irreducible characters including $1_{G}$ and hence $e=4$. Thus the degrees of the nonprincipal ordinary irreducible characters of $B_{1}(p)$ are 4,6 or 9 and the nonprincipal Brauer characters have degrees $\geqq 3$. The former fact implies that $\chi_{1}$ and $\chi_{2}$ are modular reducible so the tree having five vertices is a straight line. This yields easily

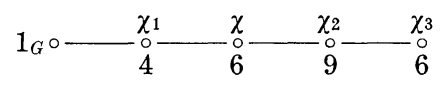

and we obtain tree (4). Now let $p=7$ so that $e=2,3$ or 6 . Clearly neither $\chi_{1}$ nor $\chi_{2}$ can be modular irreducible so the tree has at least five vertices and hence $e=6$. Thus the degrees of the nonprincipal 
ordinary irreducible characters of $B_{1}(p)$ are 6,8 or 13 and the nonprincipal Brauer characters have degrees $\geqq 4$. This latter fact implies that neither $\chi_{1}$ nor $\chi_{2}$ can have degree 6 and hence $\chi_{1}(1)=\chi_{2}(1)=13$. Now at least one of $\chi_{1}$ or $\chi_{2}$, say $\chi_{1}$, is not adjacent to $1_{G}$. Hence all characters adjacent to $\chi_{1}$ have degree $p+1$ and as we have seen these are either modular irreducible or have constituents of degree 4. This shows that all modular constituents of $\chi_{1}$ have degree divisible by 4 , a contradiction since $\chi_{1}(1)=13$. Thus this case does not occur. It now clearly suffices to assume for the remainder of this step that all $\chi \in B_{1}(p)$ with $\chi(1)=p+1$ are modular irreducible.

Let $\theta$ denote an exceptional character of $B_{1}(p)$ and we consider the possible branches leaving the vertex associated with $\theta$. Suppose first that $\theta(1) \equiv+e(p)$. The above implies easily that we can only have

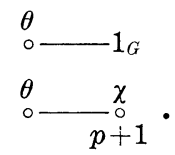

Now the first branch must occur precisely once and let the second branch occur $a$ times. Since the tree has $e$ edges we have

$$
\begin{aligned}
1+a & =e \\
1+a(p+1) & =\theta(1) .
\end{aligned}
$$

Now $e \geqq 2$ so $a \geqq 1$ and hence $\theta(1)>p$. Thus $\theta(1)=p+e$ and we obtain $a=1, e=2$ and this is tree (1).

Now let $\theta(1) \equiv-e(p)$ so that $\theta(1)=2 p-e$. Using the above information and the alternating nature of the tree we see easily that the only possible branches leaving the vertex associated with $\theta$ are

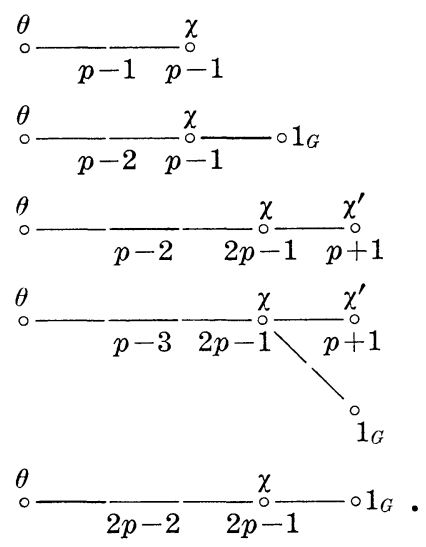

If the last branch occurs then since $2 p-e=\theta(1) \geqq 2 p-2$ we have $e=2$ and this is tree (2). Thus we can assume that only the first 
four branches occur say with multiplicities $a, b, c, d$ respectively. Since there are precisely $e$ edges in the tree we have

$$
\begin{aligned}
a+2 b+2 c+3 d & =e \\
a(p-1)+b(p-2)+c(p-2)+d(p-3) & =\theta(1)=2 p-e .
\end{aligned}
$$

Adding these two and dividing by $p$ yields

$$
a+b+c+d=2 .
$$

In addition the vertex of $1_{G}$ occurs precisely once so $b+d=1$. Thus $a+c=1$ and there are four possibilities which are easily seen to be trees (3), (4), (5) and (6).

Step 2. Let $N=N(P)$. We consider the restriction of the ordinary irreducible characters of $G$ to $N$.

Now $N=P E$ is a Frobenius group of order pe with $E=\langle x\rangle$ cyclic of order $e$. $N$ has precisely $e$ linear characters, namely those of $N / P \cong E$, and the remaining irreducible characters have degree $e$. Let $\Delta$ denote any sum of irreducible characters of $N$ of degree $e$. Clearly $\Delta_{E}=\Delta(1) / e \cdot \rho_{E}$ where $\rho_{E}$ is the regular character of $E$. This yields easily

$$
\begin{aligned}
\Delta(x) & =0 \\
\operatorname{det} \Delta(x) & =\left\{\begin{array}{cl}
1 & \text { if } e \text { is odd } \\
(-1)^{\Delta(1) / e} & \text { if } e \text { is even . }
\end{array}\right.
\end{aligned}
$$

If $e$ is even let $\delta$ denote the linear character of $N$ given by $\delta(x)=-1$.

Let $\psi$ be an ordinary irreducible character of $G$ with $\psi \notin B_{1}(p)$. Since $P$ is self centralizing it follows that $B_{1}(p)$ is the unique $p$-block of positive defect and hence $\psi$ belongs to a block of defect 0 . Thus $p \mid \psi(1)$. Since $G$ has r.b. $(2 p-1)$ this yields $\psi(1)=p$ and clearly $\psi_{P}=\rho_{P}$. Thus $\psi_{N}=\lambda+\Delta$ where $\lambda$ is linear and $\Delta(1)=p-1$. Now $G$ is simple so the linear character det $\psi$ must be principal and hence $1=\operatorname{det} \psi(x)=\lambda(x) \operatorname{det} \Delta(x)$. This yields by the above

$$
\psi_{N}=\left\{\begin{aligned}
1_{N}+\Delta & \text { if } e \text { is odd } \\
1_{N}+\Delta & \text { if } e \text { is even and }(p-1) / e \text { is even } \\
\delta+\Delta & \text { if } e \text { is even and }(p-1) / e \text { is odd } .
\end{aligned}\right.
$$

Now let $\chi \in B_{1}(p)$ and let $m(\chi)$ denote the number of linear charaters counting multiplicities which occur in $\chi_{N}$. Obviously $m(\chi)$ is the multiplicity of $1_{P}$ in $\chi_{P}$. Suppose first that $\chi_{i}, \chi_{j}$ are nonexceptional ordinary irreducible characters of $B_{1}(p)$ which are adjacent in the tree. Then $\chi_{i}+\chi_{j}=\Phi$ is a projective character and since $\Phi_{P}=\Phi(1) / p \cdot \rho_{P}$ we have easily

$$
m\left(\chi_{i}\right)+m\left(\chi_{j}\right)=\left[\chi_{i}(1)+\chi_{j}(1)\right] / p .
$$


Now suppose that $\chi_{i}$ is adjacent to the vertex of the exceptional characters $\left\{\theta_{k}\right\}$. Clearly $m\left(\theta_{r}\right)=m\left(\theta_{s}\right)$ for all $r, s$ so since $\chi_{i}+\sum_{k=1}^{(p-1) / e} \theta_{k}=\Phi$ we have with $\theta=\theta_{1}$

$$
m\left(\chi_{i}\right)+(p-1) / e \cdot m(\theta)=\left[\chi_{i}(1)+(p-1) / e \cdot \theta(1)\right] / p .
$$

Using the above two equations, the fact that the tree of $B_{1}(p)$ is connected and the obvious fact that $m\left(1_{G}\right)=1$ we obtain easily for irreducible $\chi$

$$
m(\chi)= \begin{cases}k-1 & \text { if } \chi(1)=k p-1 \\ k & \text { if } \chi(1)=k p \pm e \\ k+1 & \text { if } \chi(1)=k p+1\end{cases}
$$

There is of course additional information available, for example the fact that det $\chi(x)=1$ and the position of $\chi$ in the tree, which further limits the structure of $\chi_{N}$.

Step 3. If tree (1) occurs in step 1 , then $p$ is a Mersenne prime and $G \cong P S L(2, p+1)$.

By assumption $e=2$ and the tree of $B_{1}(p)$ has the form

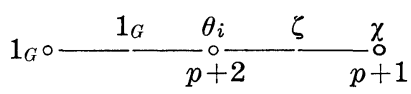

where $\theta_{i}$ represents the $(p-1) / 2$ exceptional characters with $\theta=\theta_{1}$. Let $\left\{\psi_{j} \mid j=1,2, \cdots, k\right\}$ denote the set of irreducible characters of $G$ not in $B_{1}(p)$.

Let $\alpha$ denote the unique nonprincipal linear character of $N$. By (1) and (2) we have

$$
\begin{array}{lll}
\psi_{j N}=1_{N}+\Delta_{j}^{\prime}, & \psi_{j}(x)=1 & \text { for } p \equiv 1(4) \\
\psi_{j N}=\alpha+\Delta_{j}^{\prime}, & \psi_{j}(x)=-1 & \text { for } p \equiv 3(4) .
\end{array}
$$

By (3), $\theta_{i N}=\lambda+\Delta_{i}$ and since the $\theta_{i}$ are all algebraically conjugate $\lambda$ is the same for all $i$. Thus $\operatorname{det} \theta_{i}(x)=1$ and equation (1) yield

$$
\begin{array}{lll}
\theta_{i N}=\alpha+\Delta_{i}, & \theta_{i}(x)=-1 & \text { for } p \equiv 1 \\
\theta_{i N}=1_{N}+\Delta_{i}, & \theta_{i}(x)=1 & \text { for } p \equiv 3
\end{array}
$$

Now $\chi_{N}=a 1_{N}+b \alpha+\Delta$ with $a+b=2$ by (3) and since $x$ is a $p^{\prime}$ element $a-b=\chi(x)=\zeta(x)=\theta(x)-1$. Thus (5) yields

$$
\begin{aligned}
& \chi_{N}=2 \alpha+\Delta, \quad \chi(x)=-2 \text { for } p \equiv 1 \text { (4) } \\
& \chi_{N}=1_{N}+\alpha+\Delta, \quad \chi(x)=0 \quad \text { for } p=3 \text { (4). }
\end{aligned}
$$

Equations (4), (5) and (6) and Frobenius reciprocity now yield 
( 7 )

$$
\left(1_{N}\right)^{G}=1_{G}+\sum_{1}^{k} \psi_{j}, \quad \alpha^{G}=2 \chi+\sum_{1}^{(p-1) / 2} \theta_{i} \text { for } p \equiv 1
$$

$$
\left(1_{N}\right)^{G}=1_{G}+\chi+\sum_{1}^{(p-1) / 2} \theta_{i}, \quad \alpha^{G}=\chi+\sum_{1}^{k} \psi_{j} \quad \text { for } p \equiv 3(4) .
$$

Thus since $\left(1_{N}\right)^{G}(1)=\alpha^{G}(1)=[G: N]$ and $|N|=2 p$ we obtain easily

$$
\begin{array}{lll}
|G|=p\left(p^{2}+5 p+2\right), & k=(p+5) / 2 \quad \text { for } p \equiv 1(4) \\
|G|=p(p+1)(p+2), & k=(p+1) / 2 & \text { for } p \equiv 3(4) .
\end{array}
$$

Now using $|\boldsymbol{C}(x)|=\sum \eta(x) \overline{\eta(x)}$, where $\eta$ runs over all ordinary irreducible characters of $G$, along with equations (4), (5), (6) and (8) we have

$$
\begin{array}{ll}
|C(x)|=p+7 & \text { for } p \equiv 1(4) \\
|C(x)|=p+1 & \text { for } p \equiv 3(4) .
\end{array}
$$

Since $(p+7) \nmid p\left(p^{2}+5 p+2\right)$ the case $p \equiv 1(4)$ is eliminated. Thus $p \equiv 3(4)$.

Set $S=C(x)$ so that $|S|=p+1$ and $[G: S]=p(p+2)$. We consider $\left(1_{S}\right)^{G}$. Since this character is rational and the $\theta_{i}$ are algebraically conjugate we have

$$
\left(1_{S}\right)^{G}=1_{G}+a \sum_{1}^{(p-1) / 2} \theta_{i}+b \chi+\sum_{1}^{k} c_{j} \psi_{j} .
$$

Set $c=\sum_{1}^{k} c_{j}$. By considering degrees we have

$$
p(p+2)=1+a(p+2)(p-1) / 2+b(p+1)+c p
$$

and evaluating at $x$ yields

$$
0<\left(1_{S}\right)^{G}(x)=1+a(p-1) / 2-c
$$

by (4), (5), (6) and the fact that $x \in S$. Certainly $a \leqq 2$. Also $b \leqq$ $\chi(1) / 2=(p+1) / 2$ by Frobenius reciprocity and the fact that $\chi(x)=0$. Thus $a=0$ yields a contradiction. If $a=1$ then $b \equiv 0(p)$ so $b=0$ and $c=(p+3) / 2>1+a(p-1) / 2$ again a contradiction. Thus $a=2$ and we have easily

$$
\left(1_{S}\right)^{G}=1_{G}+2 \sum_{1}^{(p-1) / 2} \theta_{i}+\chi
$$

so $\left(1_{S}\right)^{G}(x)=p$ by (5) and (6). By definition of induced character and the fact that $S=C(x)$ this implies that $S$ contains precisely $p$ distinct conjugates of $x$. Since $|S|=p+1$ this shows that $S$ is an elementary abelian 2-group and therefore that $S$ is a Sylow 2-subgroup of $G$ and $p$ is a Mersenne prime. By Burnside's lemma the nonidentity elements of $S$ are all conjugate in $N(S)$ so $N(S)>S$. 
Set $H=N(S)>S$. Then $\left(1_{H}\right)^{G}$ is a national constituent of $\left(1_{S}\right)^{G}$ and $\left(1_{H}\right)^{G}(1) \leqq p(p+2) / 3$. Thus by (10) we have easily

$$
\left(1_{H}\right)^{G}=1_{G}+\chi \cdot
$$

Therefore $G$ is a doubly transitive permutation group on the set $\Omega$ where $H=G_{\infty}$ for some point $\infty \in \Omega$. By (10) $\chi_{s}$ contains $1_{S}$ with multiplicity one so (11) implies that $S$ has two orbits on $\Omega$. Hence since $|S|=|\Omega|-1, S$ is in fact a regular normal subgroup of $G_{\infty}$. Now $|H|=p(p+1)$ so if $\widetilde{P}$ is a Sylow $p$-subgroup of $H$, then since $\widetilde{P}$ is self centralizing and $|\widetilde{P}|=|S|-1$ we see that $G$ is sharply 3transitive.

With the structure of $H$ as given above we can clearly identify $\Omega$ with $G F(p+1) \cup\{\infty\}$ in such a way that $S$ is the set of translations $\left\{\left(\begin{array}{c}z \\ z+r\end{array}\right) \mid r \in G F(p+1)\right\}$ and $\widetilde{P}$ is the set $\left\{\left(\begin{array}{c}z \\ s z\end{array}\right) \mid s \in G F(p+1), s \neq 0\right\}$. Let $\widetilde{x} \in G$ with $\tilde{x}=(0 \infty)(1) \cdots$. Then $\tilde{x}$ has order 2 and normalizes $\widetilde{P}=G_{\infty 0}$ so $\widetilde{x}$ acts in a dihedral manner on $\widetilde{P}$. If $\widetilde{x}=\left(\begin{array}{c}z \\ f(z)\end{array}\right)$ then for all $s \in G F(p+1), s \neq 0$

$$
\left(\begin{array}{c}
z \\
s z
\end{array}\right)\left(\begin{array}{c}
z \\
f(z)
\end{array}\right)=\left(\begin{array}{c}
z \\
f(z)
\end{array}\right)\left(\begin{array}{c}
z \\
s^{-1} z
\end{array}\right)
$$

so $f(s z)=s^{-1} f(z)$. Setting $z=1$ yields $f(s)=s^{-1}$. Thus $\widetilde{x}=\left(\begin{array}{c}z \\ 1 / z\end{array}\right)$ and since $G=\langle H, \widetilde{x}\rangle$ we have clearly $G \sqsubseteq P S L(2, p+1)$. By (8) we have in fact $G \cong P S L(2, p+1)$ and this step follows.

Step 4. Completion of the proof.

We now consider the remaining trees in turn. Let $\left\{\psi_{j} \mid j=\right.$ $1,2, \cdots, k\}$ denote the set of ordinary irreducible characters of $G$ not in $B_{1}(p)$.

Suppose first that we have tree (2). If $p=3$ this is the same as tree (1) so we assume that $p>3$. From

$$
1_{G} \circ-\frac{1_{G}}{2 p-1} \stackrel{\chi}{\chi} \frac{\zeta}{2 p-1} \stackrel{\theta_{i}}{\circ}
$$

and (3) and det $\chi(x)=1$ we have $\chi_{N}=1_{N}+\Delta$. Let $\alpha$ be the unique nonprincipal linear character of $N$ so that we have by (3) $\theta_{i_{N}}=$ $a 1_{N}+b \alpha+\Delta_{i}$ with $a+b=2$. Since $x$ is a $p^{\prime}$-element $a-b=\theta_{i}(x)=$ $\zeta(x)=\chi(x)-1=0$ so $\theta_{i N}=1_{N}+\alpha+\Delta_{i}$. Now by (2) all the $\psi_{j}$ occur in either $\left(1_{N}\right)^{G}$ or $\alpha^{G}$ depending on the parity of $(p-1) / 2$. Since $\left(1_{N}\right)^{G}(1)=\alpha^{G}(1)$, the above and Frobenius reciprocity imply that the $\psi_{j}$ occur in $\alpha^{G}$ and hence

$$
\left(1_{N}\right)^{G}=1_{G}+\chi+\sum_{1}^{(p-1) / 2} \theta_{i} .
$$


Since $|N|=2 p$ this yields $|G|=2 p\left(p^{2}+1\right)$. Now $\theta_{i}(1)|| G \mid$ so $(p-1) \mid\left(p^{2}+1\right)$ and this is easily seen to be a contradiction for $p>3$.

Now consider tree (3)

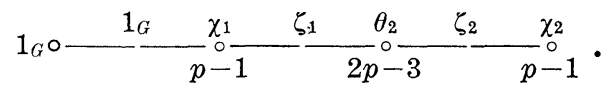

By (3), $\chi_{i N}=\Delta_{i}$ and $\chi_{i}(x)=0$ for $i=1,2$. This implies that $\zeta_{1}(x)=$ $-1, \zeta_{2}(x)=0$ so $\theta_{i}(x)=-1$. Now by $(3), \theta_{i N}=a 1_{N}+b \alpha+c \alpha^{2}+\Delta_{i}^{\prime}$ where $\alpha$ is a nonprincipal linear character and $a+b+c=2$. Since $\theta_{i}(x)=-1$ we have easily $\theta_{i N}=\alpha+\alpha^{2}+\Delta_{i}^{\prime}$. Applying Frobenius reciprocity to the above and (2) we have

$$
\left(1_{N}\right)^{G}=1_{G}+\sum_{1}^{k} \psi_{j}, \quad \alpha^{G}=\sum_{1}^{(p-1) / 3} \theta_{i}
$$

and this yields easily

$$
|G|=p(p-1)(2 p-3), \quad k=(2 p-5) / 3 .
$$

Using $|\boldsymbol{C}(x)|=\sum \bar{\eta}(x) \eta(x)$ along with the above and (2) we obtain $|\boldsymbol{C}(x)|=$ $p-1$. Now clearly $x$ is a real element so $\left|C^{*}(x)\right|=2(p-1)$ where $C^{*}(x)=\left\{g \in G \mid x^{g}=x\right.$ or $\left.x^{-1}\right\}$. Since $2(p-1)$ does not divide $|G|$ as given above, it follows that this tree does not occur.

Suppose tree (4) or (5) occurred. Since $\chi_{1}(1)=p-1$ and $\operatorname{det} \chi_{1}(x)=$ 1 , (1) and (3) imply that $(p-1) / 4$ is even. Hence by $(2), \psi_{j N}=$ $1_{N}+\Delta_{j}$. Now there are four linear characters of $N$ and at most two occur in $\theta_{N}$ so choose $\alpha \neq 1_{N}$ such that $\alpha$ does not occur in $\theta_{N}$. Thus $\alpha$ can occur only in $\chi_{2 . v}$ or $\chi_{3 . v}$ with multiplicity at most two. Hence

$$
[G: N]=\alpha^{G}(1) \leqq 2 \chi_{2}(1)+2 \chi_{3}(1)=6 p .
$$

Now choose $\beta$ so that $\beta$ occurs in $\theta_{N}$. Then

$$
[G: N]=\beta^{G}(1) \geqq \sum_{1}^{(p-1) / 4} \theta_{i}(1)=(2 p-4)(p-1) / 4 .
$$

Since $(p-1) / 4$ is even and $(p-1) / 4 \neq 2$ we have $(p-1) / 4 \geqq 4, p \geqq 17$ and

$$
6 p \geqq \alpha^{G}(1)=\beta^{G}(1) \geqq 4(2 p-4),
$$

a contradiction.

Finally consider tree (6). By (1), (2) and (3) we have easily $\chi_{1 N}=1_{N}+\Delta, \chi_{2 N}=1_{N} \Delta^{\prime}$, and $\psi_{j N}=1_{N}+\Delta_{j}$. Now since $e=5$ and $m(\theta)=2$ we can choose a linear character $\alpha$ of $N$ with $\alpha \neq 1_{N}$ and such that $\alpha$ does not occur in $\theta_{N}$. Hence by the above and the fact that $m\left(\chi_{3}\right)=m\left(\chi_{4}\right)=2$ we have $\alpha^{G}=a \chi_{3}+b \chi_{4}$ with $a, b \leqq 2$. Thus since

$$
[G: N]=\alpha^{G}(1)=a \chi_{3}(1)+b \chi_{4}(1)=(a+b)(p+1)
$$


and $[G: N] \equiv 1(p)$ we have $[G: N]=p+1$. Now choose $\beta$ so that $\beta$ occurs in $\theta_{N}$. Then

$$
p+1=[G: N]=\beta^{G}(1) \geqq \sum_{\mathbf{1}}^{(p-1) / 5} \theta_{i}(1)=(2 p-5)(p-1) / 5,
$$

a contradiction since $5 \mid(p-1)$ implies that $p \geqq 11$. This therefore completes the proof of the theorem.

Finally we consider the remaining groups with r.b. $(2 p-1)$.

THEOREM 7. Let $p$ be a prime and let $G$ be a group with r.b. $(2 p-1)$. Then we have one of the following.

(i) $G$ has a normal abelian Sylow p-subgroup.

(ii) $G$ is solvable and has p-length 1.

(iii) $G / Z(G) \cong P S L(2, p)$ or $P G L(2, p)$ for $p>3$.

(iv) $G / Z(G) \cong P S L(2, p-1)$ for $p$ a Fermat prime, $p>3$.

(v) $G / Z(G) \cong P S L(2, p+1)$ for $p$ a Mersenne prime.

(vi) $G / Z(G) \cong \operatorname{Sym}(4)$ for $p=2$.

Proof. If $p=2$ then $G$ has r.b.3. Thus by Corollary 6.5 of [8], $G$ satisfies (ii) or (vi) above. Now let $p>2$. Since $2 p-1 \leqq p^{3 / 2}$, Theorem 5 implies that $p^{2} \nmid\left|G / O_{p}(G)\right|$. With this additional fact it is easy to see that the proof of the main theorem of [6] applies also to groups with r.b. $(2 p-1)$ with $p>2$ yielding the same conclusion. (The $p>2$ assumption is used crucially in the last paragraph of the proof of Proposition 3.1 of [6].) Thus either $G$ satisfies (i) or (ii) above or $G=P_{1} \times G_{1}$ where $P_{1}$ is an abelian $p$-group and $p^{2} \nmid\left|G_{1}\right|$. Clearly $G_{1}$ has r.b. $(2 p-1)$ and if $G_{1}$ satisfies any of the above then so does $G$. Therefore it suffices to assume that $G=G_{1}$ or equivalently that $p^{2} \nmid|G|$. We assume now that $G$ does not satisfy (i). This of course implies that $p|| G \mid$.

Let $K=\boldsymbol{O}_{p^{\prime}}(G)$ and let $H / K$ be a minimal normal subgroup of $G / K$. Then $p|| H / K \mid$ and since $p^{2} \nmid|G / K|$ this implies that $H / K$ is the unique minimal normal subgroup. Now $H / K$ is a product of isomorphic simple groups and $p^{2} \nmid|H / K|$ so $H / K$ is simple. If $|H / K|=p$ then $G$ is $p$-solvable of $p$-length 1 . Thus since $G$ does not have a normal Sylow $p$-subgroup, Proposition 2.3 of [6] implies that $G$ is solvable and $G$ satisfies (ii). Hence it suffices to assume that $\bar{H}=H / K$ is a nonabelian simple group. It is convenient to first consider the possibility $p \geqq 5$.

Since $\bar{H}$ is the unique minimal normal subgroup of $\bar{G}=G / K$ we have $C_{\bar{G}}(\bar{H})=\langle 1\rangle$ and thus $\bar{G} \sqsubseteq$ Aut $\bar{H}$. Suppose $\bar{T}$ is a subgroup of $\bar{H}$ with $1<[\bar{H}: \bar{T}]<2 p$. Since $\bar{H}$ is simple and $p|| \bar{H} \mid$ we cannot have $[\bar{H}: \bar{T}]<p$. Thus $p \leqq[\bar{H}: \bar{T}]<2 p$ and $\bar{T}$ is maximal in $\bar{H}$ and hence self normalizing. If $\bar{T}$ were abelian it would follow easily that 
$\bar{T}$ is a T.I. set and then $\bar{H}$ is a simple Frobenius group, a contradiction. Thus $\bar{T}$ is nonabelian.

Let $\psi$ be an irreducible character of $K$ and let $\chi$ be an irreducible constituent of $\psi^{H}$. If $e=\left[\chi_{K}, \psi\right]_{K}$ then $\chi(1)=e t \psi(1)$ where $t=[H: T]$ and $T$ is the inertial group of $\psi$ in $H$. Suppose $T<H$ and set $\bar{T}=$ $T / K$. Since $\chi(1)<2 p$ we have $t<2 p$ and thus by the remarks of the preceding paragraph $t \geqq p$ and $\bar{T}$ is nonabelian. Thus we have $2 p>$ $\chi(1)=e t \psi(1) \geqq e p \psi(1)$ so $e=\psi(1)=1$. Now there exists an irreducible character $\eta$ of $T$ with $\eta^{H}=\chi$ and $\eta_{K}=e \psi=\psi$. Since $\bar{T}$ is nonabelian we can choose a nonlinear irreducible character $\beta$ of $T$ containing $K$ in its kernel. Thus since $\eta$ is linear, $\eta_{0}=\eta \beta$ is also an irreducible character of $T$. Let $\chi_{0}$ be an irreducible constituent of $\eta_{0}^{H}$. Then $\beta(1) \psi=\eta_{0 K}$ occurs in $\chi_{0 K}$ and therefore $\left[\chi_{0 K}, \psi\right] \geqq \beta(1)>1$. The above reasoning applied to $\chi_{0}$ now yields a contradiction. Thus $H=T$ and $H$ fixes all irreducible characters of $K$. By Brauer's lemma, $H$ fixes all conjugacy classes of $K$. Let $P$ be a Sylow $p$-subgroup of $H$. Then $P$ fixes each class of $K$ and since $K$ is a $p^{\prime}$-group, $P$ centralizes $K$. Thus if $C=C_{H}(K)$ then $K C>K$ and since $H / K$ is simple we have $H=K C$. Now $C /(C \cap K) \cong \bar{H}$ and $Z(C) \supseteqq C \cap K$ so $Z(C)=C \cap K$.

Let $D$ denote the last term in the derived series of $C$. Then clearly $D=D^{\prime}, D / Z(D) \cong \bar{H}$ and $Z=Z(D)=D \cap K$. Thus $Z$ is a homomorphic image of the Schur multiplier of $\bar{H}$. By Theorem 6, $\bar{H} \cong P S L(2, p), P S L(2, p-1)$ for $p$ a Fermat prime or $P S L(2, p+1)$ for $p$ a Mersenne prime. We have by assumption $p \geqq 5$. Also for $p=5$, $P S L(2, p) \cong P S L(2, p-1)$ and we will view this group as $P S L(2, p)$. By [10] (Satz IX, p. 119) either $Z=\langle 1\rangle$ or $\bar{H} \cong P S L(2, p), D \cong S L(2$, $p)$ and $|Z|=2$.

We show now that $K$ is central. Suppose first that $Z=\langle 1\rangle$ so that $H \cong D \times K$. Let $\chi$ be a fixed irreducible character of $D$ with $\chi(1)=p$ and let $\lambda$ be an irreducible character of $K$. Then $\chi \lambda$ is an irreducible character of $H$ so $2 p>\chi(1) \lambda(1)=p \lambda(1)$ and $\lambda(1)=1$. Thus $K$ is abelian and central in $H$. If $K$ is not central in $G$, then some linear character $\lambda$ of $K$ is not fixed by $G$. This implies easily that if $\theta$ is a constituent of $(\chi \lambda)^{G}$ then $\theta(1) \geqq 2 \chi(1)=2 p$, a contradiction. Thus $K$ is central in $G$ in this case. Now let $Z \neq\langle 1\rangle$ so that $|Z|=2$ and $D \cong S L(2, p)$. We have an epimorphism $D \times K \rightarrow D K=H$ where the kernel is the third subgroup $W$ of order 2 in the group generated by the copies of $Z$ in $D$ and $K$. Let $\lambda$ be an irreducible character of $K$. Since $Z$ is central in $K$ and $|Z|=2$ it is easy to see from the character table of $S L(2, p)([10]$, p. 128) that there exists an irreducible character $\chi$ of $D$ with $\chi(1) \geqq p$ and with $W$ in the kernel of $\chi \lambda$, an irreducible character of $D \times K$. Thus $\chi \lambda$ is a character of $H$. The preceding argument now shows first that $K$ is abelian and then that $K$ is central. We have therefore shown that $G / Z(G) \cong \bar{G}$ and it remains 
to identify $\bar{G}$.

Now $\bar{G} \sqsubseteq$ Aut $\bar{H}$ and $\bar{H}$ is a 2-dimensional projective group so the possibilities for $\bar{G}$ are given by Satz 1 of [9]. Suppose first that $\bar{H} \cong P S L(2, p)$. Then either $\bar{G} \cong P S L(2, p)$ or $\bar{G} \cong P G L(2, p)$ and we have (iii). Note the fact that $P G L(2, p)$ has r.b. $(2 p-1)$ can be seen from the character table on page 136 of [10]. We consider the remaining two cases. Thus $\bar{H} \cong P S L(2, s)$ with $2^{n}=s=p \pm 1$ and $\bar{G} / \bar{H}$ is isomorphic to a subgroup of the Galois group of $G F\left(2^{n}\right) / G F(2)$, a cyclic group of order $n$. Suppose $\bar{G}>\bar{H}$ and let $t \in \bar{G}$ correspond to a nontrivial field automorphism $x \rightarrow x^{j}$. Then in the notation of page 134 of [10], but replacing upper case by lower case letters, we have $t^{-1} a t=a^{j} \neq a$. Since $s>4$ by our assumption for $p=5$ it follows easily that $a^{j} \neq a^{-1}$ so $a^{j}$ is not conjugate to $a$ in $\bar{H}$. From the character table of $\bar{H}$ we now see easily that $t$ moves some irreducible character of $\bar{H}$ of degree $s+1$ and thus $\bar{G}$ has an irreducible character of degree at least $2(s+1) \geqq 2 p$, a contradiction. Hence $\bar{G}=\bar{H}$ and $G$ satisfies (iv) or (v). This completes the proof of the theorem for $p \geqq 5$.

Finally let $p=3$. Since $\bar{H}$ is a nonabelian simple group with r.b. $(2 p-1), \bar{H} \cong P S L(2,4) \cong P S L(2,5)$ by Theorem 6 . Certainly $G$ is not 5 -solvable and $G$ has r.b. $(2 \cdot 5-1)$. Thus by the prime 5 case already proved, $G / Z(G) \cong P S L(2,5)$ or $P G L(2,5)$. Since the latter group has an irreducible character of degree $6>2 p-1$ we have $G / Z(G) \cong P S L(2,5) \cong P S L(2, p+1)$ and $G$ satisfies (v). Thus the result follows.

\section{REFERENCES}

1. R. Brauer, Investigations on group characters, Ann. of Math. 42 (1941), 936-958. 2 . On groups whose order contains a prime to the first power I, Amer. J. Math. 64 (1942), 401-420.

3. R. Brauer and H. F. Tuan, On simple groups of finite order I, Bull Amer. Math. Soc. 51 (1945), 756-766.

4. W. Feit, Groups with a cyclic Sylow subgroup, Nagoya Math. J. 27 (1966), 571-584.

5. - On finite linear groups, J. of Algebra 5 (1967), 378-400.

6. I. M. Isaacs, Finite groups with small character degrees and large prime divisors, Pacific J. Math. 23 (1967), 273-280.

7. I. M. Issacs and D. S. Passman, A characterization of groups in terms of the degrees of their characters, Pacific J. Math. 15 (1965), 877-903.

8. - A characterization of groups in terms of the degrees of their characters II, Pacific J. Math. 24 (1968), 467-510.

9. O. Schreier and B. L. van der Waerden, Die Automorphismen der projectiven Gruppen, Abh. Math. Sem. Univ. Hamburg 6 (1928), 303-322.

10. I. Schur, Untersuchungen über die Darstellung der endlichen Gruppen durch gebrochene lineare Substitutionen, J. für Math. 132 (1907), 85-137.

Received July 24, 1968. The research of the second author was supported in part by Army Contract SAR/DA-31-124-ARO(D) 336. 


\section{PACIFIC JOURNAL OF MATHEMATICS}

\section{EDITORS}

H. ROYDEN
Stanford University
Stanford, California

\section{R. R. PHELPS}

University of Washington

Seattle, Washington 98105
J. DUGUNDJI

Department of Mathematics

University of Southern California

Los Angeles, California 90007

\section{RICHARD ARENS}

University of California

Los Angeles, California 90024

\section{ASSOCIATE EDITORS}

E. F. BeCKenbaCh
B. H. NEUMANN

F. WOLF
K. YoshidA

\section{SUPPORTING INSTITUTIONS}

\author{
UNIVERSITY OF BRITISH COLUMBIA \\ CALIFORNIA INSTITUTE OF TECHNOLOGY \\ UNIVERSITY OF CALIFORNIA \\ MONTANA STATE UNIVERSITY \\ UNIVERSITY OF NEVADA \\ NEW MEXICO STATE UNIVERSITY \\ OREGON STATE UNIVERSITY \\ UNIVERSITY OF OREGON \\ OSAKA UNIVERSITY \\ UNIVERSITY OF SOUTHERN CALIFORNIA
}

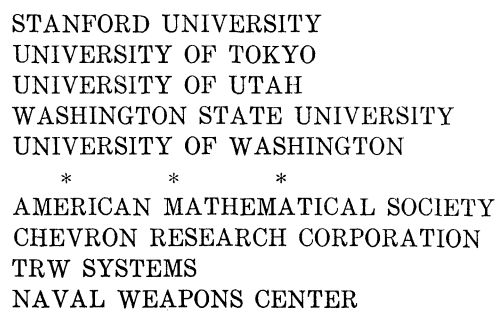

The Supporting Institutions listed above contribute to the cost of publication of this Journal, but they are not owners or publishers and have no responsibility for its content or policies.

Mathematical papers intended for publication in the Pacific Journal of Mathematics should be in typed form or offset-reproduced, double spaced with large margins. Underline Greek letters in red, German in green, and script in blue. The first paragraph or two must be capable of being used separately as a synopsis of the entire paper. It should not contain references to the bibliography. Manuscripts, in duplicate if possible, may be sent to any one of the four editors. Please classify according to the scheme of Math. Rev. 36, 1539-1546. All other communications to the editors should be addressed to the managing editor, Richard Arens, University of California, Los Angeles, California, 90024.

50 reprints are provided free for each article; additional copies may be obtained at cost in multiples of 50 .

The Pacific Journal of Mathematics is published monthly. Effective with Volume 16 the price per volume (3 numbers) is $\$ 8.00$; single issues, $\$ 3.00$. Special price for current issues to individual faculty members of supporting institutions and to individual members of the American Mathematical Society: $\$ 4.00$ per volume; single issues $\$ 1.50$. Back numbers are available.

Subscriptions, orders for back numbers, and changes of address should be sent to Pacific Journal of Mathematics, 103 Highland Boulevard, Berkeley, California, 94708.

PUBLISHED BY PACIFIC JOURNAL OF MATHEMATICS, A NON-PROFIT CORPORATION

Printed at Kokusai Bunken Insatsusha (International Academic Printing Co., Ltd.), 7-17, Fujimi 2-chome, Chiyoda-ku, Tokyo, Japan. 


\section{Pacific Journal of Mathematics \\ Vol. 29, No. $2 \quad$ June, 1969}

Bruce Langworthy Chalmers, On boundary behavior of the Bergman kernel function and related domain functionals ................... 243

William Eugene Coppage, Peirce decomposition in simple Lie-admissible power-associative rings .............................. 251

Edwin Duda, Compactness of mappings...................... 259

Earl F. Ecklund Jr., On prime divisors of the binomial coefficient......... 267

Don E. Edmondson, A modular topological lattice ............... 271

Phillip Alan Griffith, A note on a theorem of Hill ................... 279

Marcel Herzog, On finite groups with independent cyclic Sylow

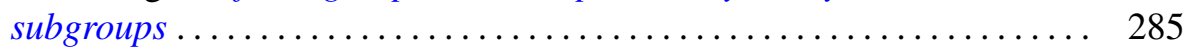

James A. Huckaba, Extensions of pseudo-valuations................. 295

S. A. Huq, Semivarieties and subfunctors of the identity functor ........ 303

I. Martin (Irving) Isaacs and Donald Steven Passman, Finite groups with small character degrees and large prime divisors. II ............ 311

Carl Kallina, A Green's function approach to perturbations of periodic

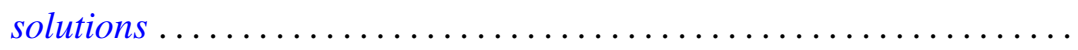

$\mathrm{Al}$ (Allen Frederick) Kelley, Jr., Analytic two-dimensional subcenter manifolds for systems with an integral ....................

Alistair H. Lachlan, Initial segments of one-one degrees ............ 351

Marion-Josephine Lim, Rank k Grassmann products ............. 367

Raymond J. McGivney and William Henry Ruckle, Multiplier algebras of

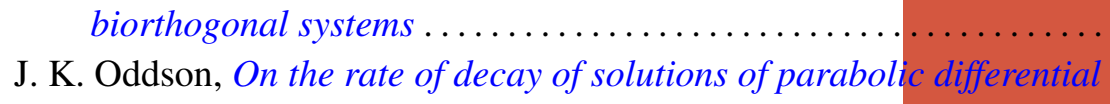

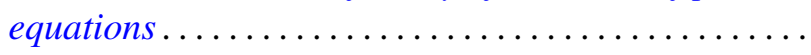

Helmut R. Salzmann, Geometries on surfaces ........... .

Annemarie Schlette, Artinian, almost abelian groups and their groups of automorphisms ............................

Edgar Lee Stout, Additional results on modules over polydisc algebras ...

Lajos Tamássy, A characteristic property of the sphere . .

Mark Lawrence Teply, Some aspects of Goldie's torsion theory. ...

Freddie Eugene Tidmore, Extremal structure of star-shaped sets ...

461

Leon Jarome Weill, Unconditional and shrinking bases in locally convex spaces... 